\title{
No clinical evidence base to support the hygiene hypothesis
}

\author{
C.P. van Schayck*, J.A. Knottnerus
}

Department of General Practice, Care and Public Health Research Institute (Caphri), University of Maastricht., P.O. Box 616, 6200 MD Maastricht, The Netherlands

\section{KEYWORDS \\ Clinical evidence; \\ Allergy; \\ Hygiene hypothesis}

\section{General practitioners and other Chealth care} providers are in receipt of conflicting information on the pathopysiology of allergy (and asthma), especially in relation to the hygiene hypothesis. It is difficult for these health care professionals to translate this information into practical advice for their patients who want to know how to avoid the development of these diseases in their children. ${ }^{1}$

\footnotetext{
*Corresponding author. Tel.: +31-433882152; fax: +31-433884225.

E-mail address: O.vanSchayck@caphri.unimaas.nl (C.P. van Schayck).

${ }^{1}$ Part of this contribution has also been published in [8].
}

Before Strachan proposed the so called hygiene hypothesis in 1989 [1], conceptual thinking of the possibility of the prevention of the development of asthma in infancy was relatively simple: a susceptible genetic background with specific environmental stimuli will lead to the development of asthma in children. So, when environmental stimuli are sufficiently reduced in these susceptible genotypes, a protection against the development of asthma would be the result.

However, after the hygiene hypothesis had been formulated, life is not that simple any more. This hypothesis postulates a protective effect of specific environmental or infection factors. The hygiene 
What evidence do we have for the hygiene hypothesis?

Several research reports suggest that:

- infections due to a sibling effect (more brothers or sisters) protect against asthma [4];

- infections due to day care protect against allergy and asthma [5];

- microbial products due to farming protect against allergy and asthma [6];

- inhalant allergens due to cats and dogs protect against allergy [7];

- breast feeding induces allergy and asthma [2].

hypothesis suggests that development of allergy (or asthma) can be prevented by a shift from T-helper cell 2 (Th-2) dominance to Th-1 dominance, induced by exposure to immune stimulants such as viruses, bacteria and endotoxins, in particular in the prenatal period or early childhood. It is interesting that the hygiene hypothesis is more and more accepted as explanation for the increase in allergy and asthma in the western world in the last decade $[2,3]$.

What actual evidence do we have for the hygiene hypothesis? Several studies are frequently cited to support it $([2,4-7]$ see Box $)$.

Is the literature indeed consistent that these stimuli are likely to reduce the development of asthma? Given the possibly huge practical implications of the hygiene hypothesis, we have to critically consider the state of the current evidence. Could there be other explanations for these observations which support the hygiene hypothesis? At this point, it is important to recognise that all in dications in favour of the hypothesis are based on observational (cross-sectional and cohort) studies and not on observations in randomised controlled interyentiom studies [8]. The general problem with cross-sectional studies is that no causal relationships can be assessed with sufficient validity. There are also validity problems with cohort studies, as the exposure is not determined by chance (by means of randomisation) and could be influenced by behaviour. And behaviour might well be a confounder, since it can be associated with both exposure and outcome. For instance, could the behaviour of parents confound the sibling and day care attendance effect? If the first two children in a family have asthma-related morbidity, the parents may not be encouraged to have more children if they fear that these might also be asthmatic, or if they feel already too heavily burdened by having two asthmatic children. This might suggest a negative association between family size and occurrence of astma in children. Also, a child susceptible for respiratory morbidity might result in parents being somewhat reluctant to put a child into day care when compared to those with non-susceptible child. In particular, parents with asthma might be afraid that their child would be exposed to infections, as they are likely to have experienced the effects that these infections can have on their own health. Until recently these asthmatic parents were also advised by their GP or asthma nurse to avoid this exposure as much as possible.

Could a healthy worker effect play a confounding role in the negative association between exposure to microbial products due to farming and the occurrence of asthma? Indeed, Vogelzang et al. [9] observed that allergic adolescents are less likely to choose a farming profession than non-allergic adolescents. Might allergic parents and parents of children with respiratory symptoms be more likely to decide not to have a cat or dog in their households than other parents? Allergic parents may have experienced what effects these pets have on their own health, and parents of susceptible children can take the precaution of avoiding pets. Indeed behaviour of parents is likely to play an important role in the exposure of high-risk children to allergens. In the PIAMA study, a RCT investigating the efficacy of allergen reducing exposure, the allergen load before the intervention measures was so low that according to the authors this is likely to be caused by increased public awareness of the potential adverse effect of allergen exposure in particular among atopic families [10]. Moreover, in a large study of more than 14.000 families it was concluded that pet-keeping seemed to be protective for the development of allergy, but this was mainly due to the fact that parents avoided exposing their child to pets as they believed that this is a risk factor for allergens [11]. And finally, could allergic mothers more likely decide to breast-fed than non-allergic mothers? They could indeed, if they are informed that this would be better for their children, so that being exposed to non-breastfeeding may seem to be correlated with better outcomes. And we know that these parents were often given advice of this sort until very recently. So, in all these cases of an observed negative relation between the exposure and the occurrence of allergy and asthma could be an effect of susceptibility eventually influencing exposure, instead of exposure reducing asthma occurrence.

In each of the quoted studies [2,4-7] attempts were made to control for confounding behaviour in 
multivariable analyses, but one has to consider that correction for confounding cannot always exclude confounding, since, for example, unknown selection phenomena and/or inappropriately measured confounding factors cannot be adjusted for post hoc.

There is nothing wrong with proposing a hypothesis and investigating it. On the contrary, that is what science is all about. But the problem is that, without being appropriately tested in well designed prospective research, the hygiene hypothesis has currently already become so popular in the news media that an increased reporting and diagnosis of asthma might even be induced as a result of an increased exposure. First, proper randomised controlled trials should be performed in which not behaviour but chance (i.e. randomisation) determines differences in exposure to environmental stimuli. Of course, controlled trials randomising day care attendance, family size, or farming profession are not very realistic to perform, but it is possible to randomise interventions to reduce infections or exposure to inhalant or feeding allergens. As to the effect of exposure on the development of asthma in infancy, the proof of the pudding will lie in controlled trials with randomised reduction of allergen exposure in birth cohorts. There are many ongoing randomised clinical trials but only a few of these with sufficient follow-up have thus far been published: the Isle of Wight study [12], a Canadian multifaceted intervention program in high-risk infants [13] and the NAC-MAAS study [14]. These three studies all investigated high-risk children (according to parental atopic status) and allocated them randomly to environmental manipulation in which several combined measures to reduce prenatal and postnatal allergen exposure were undertaken. They have shown that the reduction in allergen exposure reduces the development of allergy and/or asthma, ? and do not yield any evidence to support the hygiene hypothesis. As the currently available evidence to support the hygiene hypothesis is poor indeed, the hypothesis sometimes seems to have become faith or ideology. Scientists should take care that their messages to the general public would not go beyond or even be in conflict with present evidence. One should not suggest, for example, that eating dirt or to refuse participation in vaccination programs to prevent infectious disease would be good for children, which might be seen as ultimate consequence of the hygiene hypothesis [15]. Such advice would obviously be dangerous and can result in opposite effects. Clearly, those who consider the hypothesis as promising and to be of possible added value for practice, have the responsibility to provide valid evidence to support or reject it, before any advice should go to the public. Moreover, these scientists should take care that their messages are really evidence-based before they are translated to the daily care provided by general practitioners and other health care professionals.

At the same time, a more general reappraisal is necessary. While the hygiene hypothesis tries to cover a broad range of heterogeneous infectious and allergenic exposures, mechanisms and effects, if present, might vary across these. Specific mechanisms and effects, within the context of the complex relations between the various exposures and clinical outcomes, should be demonstrated and clarified before accepting new comprehensive theories. Accordingly, at this stage, investigations are needed focusing on specific, well targeted research questions, instead of using the very general and almost ideological term 'hygiene hypothesis', which seems too non-specific for scientific purposes.

\section{References}

[1] Strachan DP. Hay fever, hygiene, and household size. BMJ 1989;299:1259-60.

[2] Martinez FD, Holt PG. Role of microbial burden in aetiology of allergy and asthma. Lancet 1999;354(Suppl 2):1251.

[3] Sears MR, Greene JM, Willan AR, Taylor DR, Flannery EM, Cowan JO, et al. Long-term relation between breastfeeding and development of atopy and asthma in children and young adults: a longitudinal study. Lancet 2002;360:901-7.

[4] Ball TM, Castro-Rodriguez JA, Griffith KA, Holberg CJ, Martinez FD, Wright AL. Siblings, day-care attendance, and the risk of asthma and wheezing during childhood. N Engl J Med 2000;343:538-43.

[5] Krämer U, Heinrich J, Wjst M, Wichmann HE. Age of entry to day nursery and allergy in later childhood. Lancet 1999;353:450-4.

[6] Riedler J, Braun-Fahrländer C, Eder W, Schreuer M, Waser M, Maisch $S$, et al. Exposure to farming in early life and development of asthma and allergy: a cross-sectional survey. Lancet 2001;358:1129-33.

[7] Hesselmar B, Aberg N, Aberg B, Eriksson B, Bjorksten B. Does early exposure to cat or dog protect against later allergy development? Clin Exp Allergy 1999;29:611-7.

[8] van Schayck CP, Knottnerus JA. The hygiene hypothesis: a case of confounding by behaviour? J Clin Epidimol 2004, in press.

[9] Vogelzang PFJ, van der Gulden JWJ, Tielen MJM, Folgering HTM, van Schayck CP. Health-based selection for asthma, but not for chronic bronchitis in pig farmers: an evidence-based hypothesis. Eur Respir J 1999;13:187-9.

[10] van Strien RT, Verhoeff AP, van Wijnen JH, Doekes G, de Meer GE, Brunekreef B. Der $\mathrm{p} 1$ concentrations in mattress surface and floor dust collected from infants' bedrooms. Clin Exp Allergy 1995;25:1184-9.

[11] Bornehag CG, Sundell J, Hagerhed L, Janson SD. BH Study Group. Pet-keeping in early childhood and airway. Allergy 2003;58:939-44.

[12] Arshad SH, Matthews S, Gant C, Hide DW. Effect of allergen avoidance on development of allergic disorders in infancy. Lancet 1992;339:1493-7. 
[13] Chan-Yeung M, Manfreda J, Dimich-Ward H, Ferguson A, Watson W, Becker A. A randomised controlled study on the effectiveness of a multifaceted intervention program in the primary prevention of asthma in high-risk infants. Arch Pediatr Adolesc Med 2000;154:65763.
[14] Custovic A, Simpson BM, Simpson A, Kissen P, Woodcock A. Effect of environmental manipulation in pregnancy and early life on respiratory symptoms and atopy during first year of life: a randomised trial. Lancet 2001;358:188-93.

[15] Weiss ST. Eat dirt-the hygiene hypothesis and allergic diseases. N Engl J Med 2002;347:930-1.

Available online at www.sciencedirect.com

science@Direct.

\section{Available online at http://www.thepcrj.com}

